\title{
Influence de la force de Coriolis dans la dynamique des mers
}

\section{The influence of Coriolis forces on sea currents}

\author{
PAR B. SAINT-GUILY
}

CH.AHGE DE HECHERCHES DU C.N.H.S. AU MUSÉU D'HISTOIRE NATURELLE, PARIS

\begin{abstract}
Des etudes, suivant les denx points de bue de Bjerlines, Helland-Hansen d'une part et d'Eliman d'autre part, ont montré le róle important de la force de Coriolis dans les courants marins.

D'une manière générale, l'examen de plusients nombres sans dimensions permet d'estimer l'imporlance relative des différents termes des équations de lhydrodynamique. Quelques exemples numériques mettent en évidence l'importance de la force de Coriolis dans les collrants marins. La méthode n'a pas la rigueur d'une intégration des équations complètes.
\end{abstract}

\begin{abstract}
Investigations based on the theorly of Bjerlines and Helland-Hansen and on the theory of Ekman, show the great part played by Coriolis forces in sea currents.

Generally speaking, a consideration of seberal dimensionless quantities makes it possible to estimate the relative manitude of the various lerms in the hydrodynamic equations. Several worked examples bring out the magnitude of the effect of Coriolis forces on sea currents. The method is not as rigorous as integration of the complete equations.
\end{abstract}

On sait qu'en raison de la rotation de la terre. tout corps animé d'une certaine vitesse par rapport à celle-ci subit une accélération complémentaire, qui est proportionnelle à cette vitesse relative, à la vitesse de rotation de la terre et au sinus de la latitude. Cette accélération est donc nulle à l'équateur et maximum aux pôles; elle est dirigée à $90^{\circ} \mathrm{sur}$ la droite de la vitesse relative dans l'hémisphère nord, sur la gauche dans l'hémisphère sud; elle porte le nom de Coriolis (1835), bien qu'elle ait déjà été trouvée par Laplace en 1775.

Cette force joue un rôle considérable dans la circulation océanique. Cela vient de ce que les mouvements y sont lents (vitesses et accélérations faibles), du fait qu'ils intéressent des domaines étendus (profondeurs et largeurs grandes) et aussi du fait que les frottements sont relativement faibles. Si on remonte aux sources (autres que celle de Laplace, qui concerne la théorie des marées) on voit que cette importance de la force de Coriolis dans les courants marins a été établie par des études orientées suivant deux points de vue distincts.

En premier lieu, une relation thérique établie par Bjerknes (1898) a été appliquée par HellandHansen (1903) aux courants marins. Cette relation repose sur l'hypothèse d'un mouvement lent permanent et sans frottement; elle exprime l'équilibre entre l'accélération de Coriolis et celle provenant du gradient de pression existant par suite des hétérogénéités de densité. On peut ainsi déduire la vitesse aux diverses profondeurs, du champ de densité connu à partir de mesures de la température et de la salinité. Dans quelle mesure la vitesse ainsi obtenue représente-t-elle le courant réel? Il semble qu'en général elle le représente convenablement. On a Irouvé, dans plusieurs cas où des mesures directes avaient été effectuées, une bonne concordance. Pourtant l'hé- 
térogénéité des densités n'est pas la véritable cause des courants; elle est la traduction et le résultat des actions mécaniques et thermiques imposées aux océans. Ces actions, en particulier celle du vent, ne sont pas en général permanentes. Ce courant calculé à partir du champ de densité ne représente pas le courant instantané, mais le courant moyen pendant un certain intervalle de temps.

Une deuxième ligne de recherche concerne l'étude de l'action directe du vent sur la surface de la mer; cette action complexe se traduit par une tension ou force d'entrainement en surface qui est transmise en profondeur par turbulence. Le courant de vent ainsi produit a été étudié théoriquement par Ekman (1905) à partir des hypothèses suivantes : le mouvement est permanent et l'accélération de Coriolis est équilibrée par celle du frottement. Dans le schéma d'Ekman, le frottement turbulent est représenté à l'aide d'une viscosité « virtuelle » dont le coefficient ou facteur de turbulence supposé constant est bien supérieur à celui de la viscosité proprement dite. On sait que cette représentation de la turbulence a été employée pour la première fois par Boussinesq en 1877. Les résultats auxquels conduisent cette théorie d'Eliman sont assez bien confirmés par les observations. Elle montre en particulier que la force de Coriolis n'est négligeable qu'à l'équateur et près des côtes par très petits fonds.

Dans le prolongement de cette théorie d'Ekman, il faut citer une étude de Stommel, en 1948, qui a démontré que la variation de la force de Coriolis avec la latitude était à l'origine de l'intensification relative des courants, tels que le Gulf Stream et le Kuroshio, sur le bord ouest des grands océans.

$$
*
$$

Connaissant l'action primordiale de la force de Coriolis dans les courants marins, il est intéressant de savoir apprécier au moins qualitativement l'importance relative de cette action suivant les conditions imposées aux mouvements. Il faut, pour cela, introduire dans les équations du mouvement des grandeurs sans dimension. On obtient alors des paramètres également sans dimension tels que les nombres de Reynolds, de Froude, d'Elman, de Rossby, soit en comparant entre eux les différents termes des équations de Navier-Stokes ou de Reynolds, soit en intégrant complètement les équations du mouvement. L'intégration n'est malheureusement possible que dans certains cas très particuliers, ou bien sous certaines hypothèses permettant de simplifier les équations en les linéarisant. La première méthode présente l'avantage d'éviter l'inté- gration des équations, mais elle donne des indications un peu floues et qui appellent certaines réserves. Les équations fondamentales de l'hydrodynamique peuvent s'écrire sous forme vectorielle :

$$
\begin{gathered}
(\partial \vec{u} / \partial t)+\vec{u} \cdot \overrightarrow{\operatorname{grad}} u+\vec{\lambda} \vec{u} \vec{u} \\
=-(1 / \rho) \overrightarrow{\operatorname{grad}} p+\overrightarrow{\mathrm{G}}+(u / \rho) \Delta \vec{u} \\
\text { div. } \vec{u}=0
\end{gathered}
$$

$\vec{u}$ désigne le vecteur vitesse, $\vec{\lambda}$ le vecteur rotation de la terre, $|\lambda|=2 \omega \sin \varphi$, $\omega$ la vitesse angulaire de rotation, la latitude, la densité, $p$ la pression et $\mu$ le facteur de turbulence suivant la représentation de Boussinesq; cette représentation suppose implicitement que l'on considère un mouvement moyen à des échelles d'espace et de temps convenables.

Cette équation vectorielle comprend six termes qui sont :

1. - La force d'inertic ou accélération temporelle,

2. - La force d'inertie ou accélération spatiale (non linéaire);

3. - La force de Coriolis;

4. - La force de pression;

5. - La force de gravité

6. - La force de frottement.

Les rapports de ces différents termes définissent des nombres sans dimension caractéristiques du mouvement, ainsi :

Le nombre de Boussinesq (équivalent du nombre de Reynolds):

$$
\mathfrak{B}==\frac{\text { force d'inertie spatiale }}{\text { force de frottement }}
$$

Le nombre de Froude :

$$
\mathscr{F}=\frac{\text { foree d'inerlic spatiale }}{\text { force de gravité }}
$$

Le nombre d'Euler :

$$
\mathfrak{g}=\frac{\text { force d'inertie spatiale }}{\text { force de pression }}
$$

Le nombre g :

$$
\xi=\frac{\text { Corce d'inertie temporelle }}{\text { torce d'inertie spatiale }}
$$

Le nombre d'Ekman :

$$
\mathcal{E}=\frac{\text { force de Coriolis }}{\text { force de frottement }}
$$

Le nombre de Rossby:

$$
\mathfrak{c}=\frac{\text { force d'inertie tolale }}{\text { force de Coriolis }}
$$


Cind nombres suffisent à caractériser le mouvement, par exemple les cinc premiers. On peut, bien entendu, remplacer l'un quelconque de ces nombres par le produit ou le rapport des puissances queleonques de ce nombre et de plusieurs autres.

Dans le cas des courants marins, les dimensions horizontales sont toujours très grandes devant la profondeur (rapport de lordre de mille à un); on peut done en première approximation négliger les composantes verticales de l'accélération et de la vitesse, et considérer la pression comme égale à la pression hydrostatique. On exprime celte dernière hypothèse en écrivant que le nombre de Froude est nul. On peuc de plus admettre, pour simplifier, que les hétérogénéités de densités sont représentées par une dénivellation de la surface libre.

Si l'on désigne respectivement par :

$$
u, a, h, \mathrm{~T}, \zeta / a, \rho, \mu, g, \lambda=2 \text { (1) } \sin \varphi
$$

les: vitesse, plus petite dimension horizontale du domaine considéré, profondeur, temps, pente de la surface, densité, facteur de turbulence, accélćration de la pesanteur et parametre de Coriolis, représentatifs du mouvement, on obtient les expressions suivantes des nombres adimensionmels :

$$
\begin{aligned}
& \mathcal{B}=\frac{u h^{2}}{u a} ; \quad y=\frac{u^{2}}{g \zeta} ; \quad y=\frac{a}{u \mathrm{~T}} \\
& \mathcal{G}=\frac{\operatorname{ch} h^{2}}{\mu} ; \quad \mathcal{C}=\frac{u}{\lambda a}+\frac{1}{\lambda \mathrm{T}} ; \quad \mathscr{H}=0
\end{aligned}
$$

Si le mouvement est périodique, T représente la période divisée par $2 \pi$; s'il est permanent, est nul. Le mouvement est entièrement caracté. risé par quatre de ces nombres ou par quatre combinaisons convenables de ceux-ci, et par trois seulement si gु est nul. Si l'on veut représenter des mouvements naturels sur modèles réduits, il faut en général conserver ces nombres, ce qui impose des règles de similitudes.

Voici maintenant quelques exemples de mouvements naturels qui montrent comment ces nombres sans dimension donnent une idée de l'importance relative des forces agissantes.

\section{Premier EXemple:}

Courants de marée dans une région côtière de dimensions comparables à celles de la baie du Mont Saint-Michel :

$a=30 \mathrm{~km} ; \quad h=20 \mathrm{~m} ; \quad \zeta=60 \mathrm{~cm} ; \quad \lambda=10^{-4} \mathrm{~s}^{-1}$;

$\mathrm{T}=12$ heures; $u=1,5 \mathrm{~m} / \mathrm{s} ; \mu=300 \mathrm{c.g} . \mathrm{s}$.

on a done :

$$
\mathcal{G} \sim 1 ; \quad \mathcal{C} \sim 2 ; \quad \mathcal{H} \sim 1 / 3 ; \quad \text { 皮 } \sim 3 ;
$$

les forces sont donc presque toutes du même ordre; celles d'inertie temporelle, de Coriolis, de pression et de frottement sont du même ordre et un peu plus importantes que celles d'inertie spatiale.

\section{Deuxitane EXEMPLE :}

Courants de marée dans une mer de dimensions comparables à celles de la Manche:

$\alpha=100 \mathrm{~km} ; \quad h=-70 \mathrm{~m} ; \quad \zeta=2 \mathrm{~m} ; \quad \lambda=-10^{-4} \mathrm{~s}^{-1} ;$ $\mathrm{T}=12$ henres; $\quad u=1,5 \mathrm{~m} / \mathrm{s} ; \quad u=1,510^{3} \mathrm{c} . \mathrm{g} . \mathrm{s}$. on a :

$$
\mathcal{E} \sim 3 ; \quad \mathcal{C} \sim 3 / 2 ; \quad \mathscr{J} \sim 1 / 10 ; \quad \text { g } \sim 12 .
$$

Les forces de Coriolis, d'inertie temporelle et de pression, sont donc les plus importantes; viennent ensuite les forces de frottement, puis la force d'inertie spatiale.

\section{Tronstime ExEMPre :}

Courant de pente (ou de densité) permanent dans une mer de dimensions comparables à celles de la Méditerranée occidentale :

$$
\begin{gathered}
a=1000 \mathrm{~km} ; \quad h=2000 \mathrm{~m} ; \quad \zeta=2 \mathrm{~m} ; \\
\lambda=10^{-4} \mathrm{~s}^{-1} ; \quad u=20 \mathrm{~cm} / \mathrm{s} ; \quad y=2.10^{3} \mathrm{cog} . \mathrm{s} .
\end{gathered}
$$

on a :

$$
\mathcal{E} \sim 2.10^{3} ; \quad \mathcal{C} \sim 1 / 500 ; \quad \mathscr{T} 1 / 500 ; \quad \text { 等 }=0 ;
$$

Les forces de Coriolis et de pression sont done du même ordre et beaucoup plus importantes que toutes les autres. Cet exemple correspond aux hypothèses de Bjerknes et Helland-Hansen. Avec les hypothèses d'Ekman, qui reviennent à négliger les termes d'inertie spatiale et temporelle et à supposer la pression égale à la pression hydrostatique, on peut intégrer comvlètement les équations. Mais la précision des indications que l'on peut en déduire n'est guère meilleure. Elle est limitée en particulier par le fait que le facteur de turbulence $\mu$ n'est pas en réalité une constante; quant à sa valeur moyenne, elle est connue d'une façon très approximative.

Ces exemples montrent l'importance du rôle joué par la force de Coriolis dans les courants marins. Il faut cependant remarquer que cette manière de procéder n'est pas rigoureuse comme celle qui consisterait à intégrer les équations complètes. Elle donne des ordres de grandeur utiles, mais elle peut conduire à des erreurs si le mouvement est complexe; par exemple, si dans le domaine étudié, il se produit plusieurs veines étroites de courant séparées par des contrecourants. Dans ce cas, il conviendrait de choisir 
pour dimension horizontale a la largeur de la veine de courant la plus étroite.

$$
\because:
$$

Le champ d'application des estimations de ce genre est linnité pour plusieurs raisons. En premier lieu, la représentation de la turbulence de Boussinesq substitue au mouvement réel, extrêmement complexe, un mouvement moyen virtuel Il est évident que cette simplification avant l'intégration doit jouer le rôle d'écran pour certaines caractéristiques des phénomènes réels. M. le Professeur Kravtchenko a attiré mon attention sur d'autres raisons profondes qui sont liées aux propriétés des équations aux dérivées partielles de l'hydrodynamique. Je cite simplement deux de ces raisons. Premièrement : « des causes arbitraitement petites peuvent produire des effets finis ». Dans les équations aux dérivées partielles, la présence de termes arbitrairement petits d'ordre élevé, ou de très petites variations des paramètres, peuvent changer totalement la nature et l'aspect des solutions. Ainsi il n'est pas toujours vrai que lorsque le coefficient d'un terme tend vers zéro, la solution tende vers celle qu'on obtient en annulant ce coefficient. Ce « paradoxe de limite » est illustré par la transition du régime laminaire au régime turbulent. Deuxiemement : « un probleme dont les données sont symétriques n'a pas nécessairement une solution symétrique stable ». Il faudrait donc toujours étudier la stabilité des solutions obtenues. Un exemple de ce "paradoxe de symétrie » est donné par le sillage d'un cylindre se déplaçant dans un fluide : on sait que pour un nombre de Reynolds supérieur à 50, ce sillage n'est plus symétrique, mais formé de tourbillons alternés. Il est bien clair que ces pseudo-paradoxes disparaissent, en tant que tels, dans une étude complète et exacte, c'est-à-dire, dans une étude qui tient compte de toutes les actions physiques essentielles.

Enfin, je crois qu'il convient de rappeler que la force de Coriolis et sa variation avec la latitude tendent généralement à diminuer l'instabilité. Cet effet stabilisateur de la force de Coriolis est une question très importante, mais c'est une autre et difficile histoire.

\section{IS C USSIO N}

président: M. Lacombe

M. le Président remercie M. Sant-Gurx de son exposé et donne la parole à ceux gui la désirent.

M. Kravtchexro, commentant l'exposé de M. SAINTGurry, illustre sa thérie par l'exemple de Borel : l'addition d'un terme trés petit à une équation non linéaire peut troubler très protondément la nature de la solution.

M. Bresel demande quelles sont les importances relatives des effets thermiques d'une part, et des efforts de traction tangentielle dus au vent d'autre part, sur l'organisation des grands courants oceaniques.

M. SAINT-Gunf estime qu'il faut distinguer le point de vue pratique et le point de vue causal. Il semble bien que ce soit le vent qui soit la cause essentielle de tous les courants marins. Si, dans le cas d'un mouvement moyen, on peut calculer le courant en tenant compte seulement de la répartition moyenne des pressions, c'est que l'on considère alors les variations de densite comme un effet et non comme la cause, comme le fait remarquer M. Biester.

M. Breser se demande encore, si en l'absence de vent, l'échauffement différentiel, plus élevé à l'équateur que dans les régions polaires, ne provoquerait pas une circulation analogue.

M. SAINT-Gully répond qu'il y aurait sans doute une circulation thermique, mais très faible.
M. le Président confirme cette réponse, en remarquant que la machine thermodynamique constituée par les eaux a un rendement pratiquement nul ('eau est chauffée à l'équateur sous la pression atmospluérique et refroidie dans les régions polaires aussi sous la pression atmosphérique). Mais la cause essentielle du mouvement est bien le vent et la répartition des densites, traitée par la méthode de Bjerlines, peut rendre compte du courant de vent, car celui-ci modife cette reppartition.

On peut d'ailleurs montrer que, sous réserve de schématisation, le flux d'eau total transporté entre une surface suffisamment profonde où l'eau est supposée immobile et la surface marine, est la somme du flux d'eau donné par la méthode de Bjerlnnes et d'un flux d'eau qui est transporté, par le vent, dans une mer homogène, c'est-à-dire selon le schéma d'Eliman, et ceci en tenant compte d'un facteur de turbulence de Boussinesq sur la verticale.

On peut ajouter aussi que le rappont des forces d'inertie à la force de Coriolis est égal au rapport de la période du phénomène étudié à la durée du demi-joul pendulaire, ce qui permet de voir que la force de Coriolis est négligeable pour la houle dont les périodes sont de l'ordre de la dizaine de secondes, et n'est plus négligeable pour les périodes de l'ordre de celles de la marée.

M. le Président remercie encore M. SAINT-Guily ainsí que les persomnes qui ont pris la parole. 